\title{
Resistance to Inhibitors of Dihydrofolate Reductase in Strains of Lactobacillus casei and Proteus vulgaris
}

\author{
By S. SINGER, GERTRUDE B. ELION ANd G. H. HITCHINGS \\ Wellcome Research Laboratories, Burroughs Wellcome and Co. \\ (U.S.A.) Inc., Tuckahoe, New York
}

(Received 23 April 1965)

\begin{abstract}
SUMMARY
Strains of Lactobacillus casei and Proteus vulgaris resistant to small molecule inhibitors of dihydrofolate reductase were isolated under various nutritional conditions. When thymine and/or purines were available in the media, several of the isolated strains had nutritional requirements for these metabolites, but resistant lines could still be isolated in their absence. Thus, the biochemical alteration accompanying resistance could be predetermined to a major extent by the design of the experiment.

The wild-type strain of Proteus vulgaris did not incorporate exogenous thymine, and was insensitive to thymine antagonists, while the thyminerequiring strain was highly sensitive to 5-bromouracil, dithiothymine and 2-thiothymine. This suggested that resistance was accompanied by the appearance of a permeability or transport system for thymine.
\end{abstract}

\section{INTRODUCTION}

Replicate isolates of a number of strains of Lactobacillus casei have been consistent with respect to the biochemical lesion accompanying resistance. These lesions generally have taken the form of a deletion (or major alteration) of the enzyme system dealing with the incorporation of the analogue. Thus the diaminopurine-resistant $L$. casei was unable to incorporate adenine (Elion, Singer \& Hitchings, 1953; Balis et al. 1952), the 8-azaguanine-resistant line failed to use guanine (Elion, Singer \& Hitchings, 1956) and the 6-mercaptopurine-resistant clone was unable to utilize exogenously supplied hypoxanthine (Elion et al. 1953). In these instances the analogues affect biochemical pathways which are dispensable, 'salvage' mechanisms, and the organism can obtain its requirements for growth through alternate mechanisms involving synthesis de novo.

Inhibitors of dihydrofolate reductase are in another category in this respect since the product of the inhibited reaction, tetrahydrofolate, participates, in its coenzymic forms, in a variety of essential reactions (Friedkin, 1963; Huennekens \& Osborne, 1959). Moreover, 5,10-methylenetetrahydrofolate, the coenzyme of thymidylate synthetase, is oxidized when its one-carbon fragment is transferred, and its renewal depends on the continued activity of dihydrofolate reductase.

The present paper deals with resistance to small molecular inhibitors of dihydrofolate reductase (Hitchings et al. 1952; Hitchings \& Burchall, 1965). It will be shown that nutritional requirements for the end products of enzymic systems involving tetrahydrofolate-containing coenzymes appeared concurrently with re- 
sistance. The nature of these requirements correlated closely with the composition of the medium from which the resistant isolate was obtained. Thus the outcome of the experiment designed to reveal the biochemical mechanism of resistance was predetermined to a considerable extent by the conditions of the experiment itself. Preliminary accounts of several portions of this study have been given (Singer, Elion \& Hitchings, 1957; Singer, Elion \& Hitchings, 1958; Hitchings, Singer \& Elion, 1958).

\section{METHODS}

Media. Proteus vulgaris ATcc 9920 and the mutants derived from it were grown and maintained as described by Elion, Singer \& Hitchings (1960). The basal medium (PVO) and a supplemented medium containing thymine ( $1 \mu \mathrm{g} . / \mathrm{ml} .: \mathrm{PVT})$ were employed. Growth was estimated by measurements of turbidity with a Lumetron (model 403-E) photoelectric colorimeter, adjusted so that uninoculated medium gave a zero reading and opacity was 100 .

The experiments with Lactobacillus casei (ATCC 7469) and the mutants derived from it were done as described by Hitchings et al. (1950) in basal medium $O$, plus the supplements of folic acid, thymine, adenine sulphate and drug described below. Growth after 3 days' incubation was estimated by titration of $10 \mathrm{ml}$. cultures with $0 \cdot 1 \mathrm{~N}$ sodium hydroxide. The various media used were:

OFA $=$ basal medium $(0)+$ folic acid $0.05 \mathrm{~m} \mu \mathrm{g} . / \mathrm{ml}$. (sufficient for half-maximal growth),

$\mathrm{FA}+=\mathrm{OFA}+$ folic acid $0.625 \mathrm{~m} \mu \mathrm{g} . / \mathrm{ml}$. (sufficient for maximal growth),

PFA = OFA + adenine sulphate $10 \mu \mathrm{g} . / \mathrm{ml}$.,

TFA $=$ OFA + thymine $1 \mu \mathrm{g} . / \mathrm{ml}$,

PTFA $=$ OFA + adenine sulphate $10 \mu \mathrm{g} . / \mathrm{ml}$. + thymine $1 \mu \mathrm{g} . / \mathrm{ml}$. ,

PT $=0$ +adenine sulphate $10 \mu \mathrm{g} . / \mathrm{ml}$. + thymine $1 \mu \mathrm{g} . / \mathrm{ml}$.

Isolation of the mutants. Strains of Lactobacillus casei resistant to pyrimethamine (2,4-diamino-5-p-chlorophenyl-6-ethylpyrimidine, B.W. 50-63) were isolated by the gradient plate technique (Szybalski \& Bryson, 1952) in the presence of pyrimethamine and folic acid, with or without adenine sulphate and/or thymine. Strains of Proteus vulgaris resistant to diaveridine (2,4-diamino-5-(3',4'-dimethoxybenzyl)-pyrimidine, B.W. 49-210: Roth et al. 1962) and to sulphadiazine or sulphadiazine+diaveridine were similarly obtained by means of the gradient plate technique. Except where Proteus strains resistant to various amounts of diaveridine were desired, the concentration of all the drugs used, both for the isolation and maintenance of the respective mutants, was $300 \mu \mathrm{g} . / \mathrm{ml}$. Once isolated the strains were maintained in the presence of the drug in the medium from which they were isolated. Some of the properties of the resistant strains are given in Table 1.

Thymineless death. Lactobacillus casei strain 50-63/II was inoculated into: (1) basal medium $O$, which contains all essentials except folic acid, thymine or adenine; (2) medium PTFA, a complete medium; and (3) medium PFA where folic acid and purine are supplied but thymine is omitted. Plating counts were made after $0,6,24,30,48,64$, and $72 \mathrm{hr}$.

Incorporation of $\left[2-{ }^{14} C\right]-$ thymine into bacterial $D N A$. The various strains of Proteus vulgaris were grown in replicate batches of $250 \mathrm{ml}$. of PVO medium con- 
taining [2-14 $\mathrm{C}$-thymine $(1.05 \mathrm{C} / \mathrm{mole})$. Lactobacillus casei was grown similarly in OFA medium with added thymine. The bacteria were harvested, dried and defatted (cold TCA, ethanol, ethanol + ether $(3+1)$, ether: Balis et al. 1952). The dried and defatted bacteria were digested directly with $70 \%$ perchloric acid for $1 \mathrm{hr}$ in a boiling water bath. The perchloric acid digest was then neutralized with potassium hydroxide. The thymine present in the neutralized supernatants was determined in two ways, microbiologically, using the thymine-requiring strain 50-63/II, and spectrophotometrically. For the latter purpose, the hydrolysates were subjected to chromatography in a butanol + ammonia system, the radioactive spots were eluted with $0.1 \mathrm{~N}$-hydrochloric acid and the absorption at $263 \mathrm{~m} \mu$ measured. The radioactivity was determined by means of a Nuclear Chicago Model D 47 internal gas flow counter. All counts were carried out for a time sufficient to give a probable error of less than $10 \%$. No coincidence correction was required. The results are reported in terms of relative specific activities (R.S.A.), i.e. the ratio of specific activities of the isolated and the proffered thymine.

Table 1. Properties of resistant strains

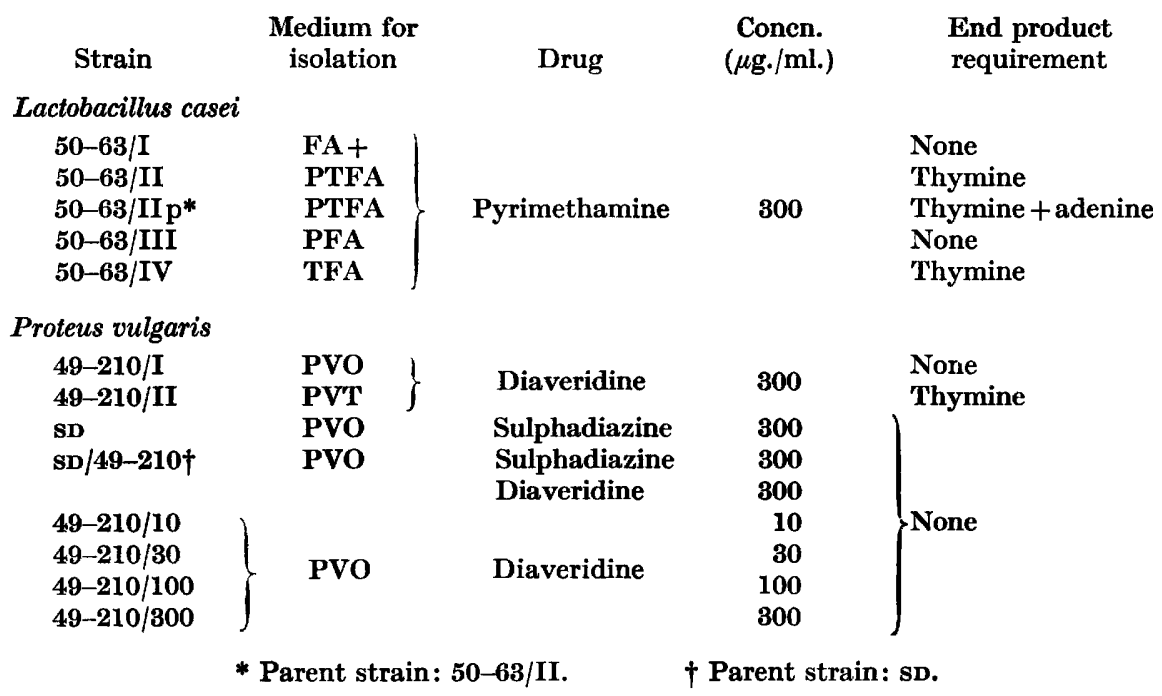

\section{RESULTS}

The wild type of Lactobacillus casei can grow in the absence of purine and thymine when folic acid is present. This is seen in Table 2 which compares the growth of the wild and resistant strains of $L$. case $i$ in various media. Strain 50-63/III grows in all of the media but somewhat more slowly than the wild strain. Strains 50-63/II and $50-63 / \mathrm{IV}$, on the other hand, have absolute requirements for thymine. Nevertheless, they synthesize purines since they grow in TFA medium from which purine is absent. When strain 50-63/II was transferred repeatedly in PFTA medium containing drug, it apparently lost its ability to synthesize adenine. This new strain, 50-63/II p, could be grown only in the presence of both purine and thymine (PT or PTFA media). Subsequent experiments have shown that guanine, hypo- 
xanthine or xanthine are able to substitute for adenine in satisfying the purine requirement of this strain.

The abilities of the wild type and strain 50-63/II to utilize thymine, its deoxyriboside and deoxyribotide, were compared (Fig. 1). The data for strains 50-63/II p and 50-63/IV are not shown, but their responses to exogenous thymine, thymidine and thymidylate, and their responses in the cross resistance experiments to be described, were identical to that of the 50-63/II strain. As can be seen, the mutant utilized thymine well but thymidine and thymidylic acid were used poorly. The wild strain utilized thymidine somewhat better but it, too, preferred free thymine.

Table 2. Growth of wild type and mutant strains of Lactobacillus casei in various media

\begin{tabular}{|c|c|c|c|c|c|c|c|}
\hline \multirow[b]{2}{*}{ Strain } & \multirow{2}{*}{$\begin{array}{l}\text { Medium for } \\
\text { isolation }\end{array}$} & \multicolumn{6}{|c|}{ Titre* } \\
\hline & & OFA & $\mathbf{F A}_{+}$ & PT & PFA & TFA & PTFA \\
\hline Wild type & - & $6 \cdot 1$ & $13 \cdot 0$ & $5 \cdot 8$ & $8 \cdot 3$ & $5 \cdot 5$ & $9 \cdot 5$ \\
\hline $50-63 / I$ & $\mathbf{F A _ { + }}$ & $1 \cdot 4$ & $6 \cdot 1$ & $1 \cdot 2$ & $3 \cdot 6$ & $1 \cdot 5$ & $1 \cdot 8$ \\
\hline $50-63 / \mathrm{II}$ & PTFA & $0 \cdot 6$ & 0.5 & $4 \cdot 7$ & $0 \cdot 6$ & 4.2 & $8 \cdot 1$ \\
\hline 50-63/IIp & PTFA & $0 \cdot 4$ & $0 \cdot 4$ & $2 \cdot 4$ & 0.4 & $0 \cdot 4$ & 8.5 \\
\hline $50-63 /$ III & PFA & $3 \cdot 6$ & $11 \cdot 6$ & 4.5 & 6.5 & $3 \cdot 7$ & $7 \cdot 5$ \\
\hline $50-63 / \mathrm{IV}$ & TFA & 0.4 & 0.5 & $5 \cdot 0$ & $0 \cdot 4$ & $9 \cdot 2$ & $10 \cdot 7$ \\
\hline
\end{tabular}

The quantity of thymine required for growth in a folic acid-free medium (PT) was the same for the two strains.

The wild type and most of the drug-resistant strains of Proteus vulgaris were able to grow well in the absence of an exogenous source of folic acid, thymine and/or purines. These growth factors neither increased the growth of these strains when added nor reversed the inhibition caused by folic acid antagonists. However, strain 49-210/II possessed an absolute requirement for thymine. The utilization of thymine, thymidine and thymidylate by this strain are shown graphically in Fig. 2. Although this strain was developed in the presence of thymine, both thymidine and thymidylate are used more efficiently than thymine. This is in contrast to the thymine-requiring strains of $L$. casei which use the free base more efficiently than the deoxyribose derivatives. Neither uracil nor cytosine could replace the thymine requirement for 49-210/II.

The Lactobacillus casei and Proteus vulgaris mutants which required thymine exhibited the phenomenon of 'unbalanced growth' and 'thymineless death' (Cohen \& Barner, 1954). This is shown by the data presented in Fig. 3. In the complete PFTA medium, the growth curve for 50-63/II reaches a plateau. In 0 medium the death rate was similar to that observed with the wild-type strain. But where only thymine was omitted (PFA medium) a precipitous death curve was observed. Microscopic examination of $72 \mathrm{hr}$ cultures of this strain grown in PFA medium showed the classical picture of unbalanced growth with long filamentous cells in PFA medium but normal coccoid rods in PTFA medium. Similar results were obtained with the other thymine-requiring mutants.

Incorporation of $\left[{ }^{2-14} \mathrm{C}\right]$-thymine into the $\mathrm{DNA}$ of various strains of Proteus vulgaris. In view of the thymine requirement of strain 49-210/II, it was decided 


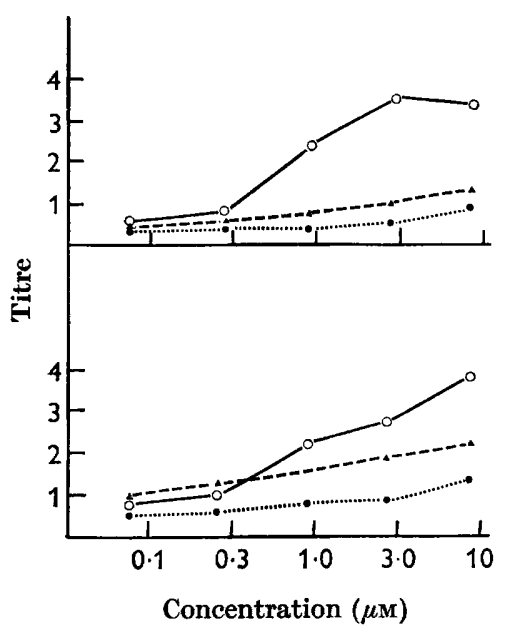

Fig. 1

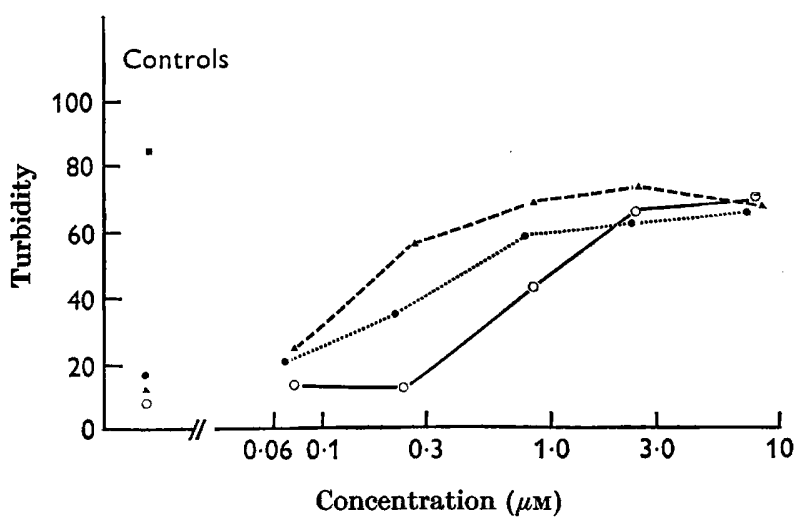

Fig. 2

Fig. 1. Growth of wild and thymineless strain of $L$. casei with thymine-containing metabolites in purine-containing medium. Upper figure, strain 50-63/II. Lower figure, wild type. $\bigcirc-O$, thymine; $\Lambda-\cdots-\Lambda$, thymidine;...-- , thymidylate. Ordinate, ml. $0 \cdot 1 \mathrm{~N}-$ acid $/ 10 \mathrm{ml}$. culture.

Fig. 2. Growth of thymineless strain of Proteus vulgaris with thymine-containing metabolites. $\mathrm{O}-\mathrm{O}$, thymine; $\Delta---\Delta$, thymidine;...--0 , thymidylate. Controls for each experiment with strain $49-210 /$ II indicated along ordinate; $n$, control growth of wild type. Ordinate, turbidity (100 less the per cent transmission).

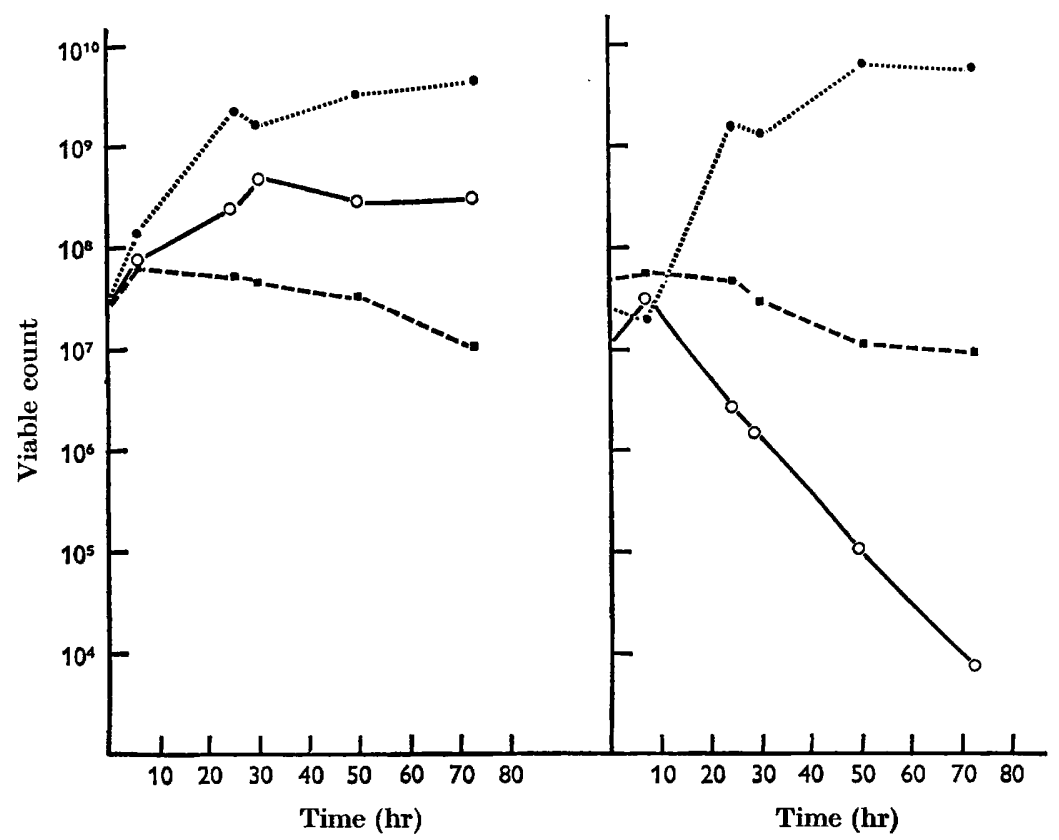

Fig. 3. Viability of wild and thymineless strains of Eactobacillus casei in various media. Figure on left, wild type; figure on right, strain 50-63/II. Media:.- .0 , PTFA; O- O, PFA; $\mathbf{a}---\mathbf{0}$, basal. Ordinate, plate count/ml. culture. 
to investigate possible alterations in the uptake of $\left[2-{ }^{14} \mathrm{C}\right]$-thymine by the Proteus strains (Table 3). Essentially no incorporation of $\left[2{ }^{14} \mathrm{C}\right]$-thymine into the DNA of wild type or strain 49-210/I was found, whereas strain 49-210/II incorporated $\left[2-{ }^{14} \mathrm{C}\right]$-thymine without change in its specific activity. In one experiment, the wild type was grown in the presence of quantities of SD and diaveridine which limited but did not entirely suppress, growth, to investigate whether the incorporation of thymine might be an expression of stress. However, no incorporation of thymine resulted. The incorporation of exogenous thymine by the wild type was concentration dependent; but complete suppression of synthesis de novo was not attained and at the highest concentration of thymine, the results were erratic and sometimes, as shown in Table 3, a lower incorporation of proffered thymine was found at a concentration of $1 \mu \mathrm{g} . / \mathrm{ml}$. than at $0.3 \mu \mathrm{g} . / \mathrm{ml}$.

Table 3. Incorporation of thymine during growth

\begin{tabular}{|c|c|c|}
\hline \multirow[b]{2}{*}{ Strain } & \multicolumn{2}{|c|}{ Thymine } \\
\hline & $\begin{array}{l}\text { Conen. } \\
(\mu \mathrm{g} \cdot / \mathrm{ml} .)\end{array}$ & $\begin{array}{r}\text { r.s.a. } \\
(\%)\end{array}$ \\
\hline Proteus, wild type & $\begin{array}{l}0.12 \\
0.12 \\
0.10\end{array}$ & $\begin{array}{l}0.09 \\
0.015 \\
0.036^{*}\end{array}$ \\
\hline $\begin{array}{r}\text { Proteus 49-210/I } \\
49-210 / I I\end{array}$ & $\begin{array}{l}0 \cdot 12 \\
0 \cdot 12\end{array}$ & $\begin{array}{c}0.08 \\
96 \cdot 7\end{array}$ \\
\hline Lactobacillus, wild type & $\begin{array}{l}0 \cdot 03 \\
0 \cdot 10 \\
0 \cdot 30 \\
1 \cdot 0 \\
0 \cdot 3\end{array}$ & $\begin{array}{c}10 \cdot 3 \\
38 \cdot 0 \\
78 \cdot 0 \\
56 \cdot 3 \\
5 \cdot 3 \dagger\end{array}$ \\
\hline
\end{tabular}

Cross-resistance. The effects of antagonists of folic acid and of end products of one-carbon metabolism on wild-type and resistant strains of Lactobacillus casei and Proteus vulgaris are shown in Tables 4 and 5 respectively. Strains 50-63/I and 50-63/II were found to be resistant to all of the folic acid antagonists tested, including representative diaminoquinazolines, pyridopyrimidines, pteridines, benzyl- and phenylpyrimidines. In contrast, Proteus strains 49-210/I and 49-210/I were as sensitive as the wild strain to pyrimethamine and the quinazoline, although resistant to benzyl-, phenoxy- and pyridopyrimidines. In addition, both strains were hypersensitive to SD. Reciprocally, the SD-resistant Proteus strain sD had an increased sensitivity to the folic acid antagonists, diaveridine and the diaminoquinazoline.

While uracil antagonists such as 6-azauracil and purine antagonists such as 6-mercaptopurine, 8-azaguanine and diaminopurine were equally effective against the wild type and resistant strains of Lactobacillus casei, their effects against Proteus strains were more varied. Both diaveridine-resistant strains were more sensitive than the wild type to 8-azaguanine and 2,6-diaminopurine. However, strain 49-210/II was no more sensitive than the wild type to 6-mercaptopurine. 
Both strain 49-210/I and 49-210/II were relatively resistant to dithiouracil, but as sensitive as the wild strain to 6-azauracil and isobarbituric acid. In contrast, strains SD and SD/49-210 were generally insensitive to all of the uracil and purine antagonists. The effects of a number of end product analogues on wild type have been reported earlier in studies designed to explore their potential for further potentiation of SD: diaveridine combinations (Elion, Singer \& Hitchings 1960).

Table 4. Cross-resistance studies with strains of Lactobacillus casei

\begin{tabular}{|c|c|c|c|c|c|c|}
\hline & & Wild tyl & & & & \\
\hline $\begin{array}{c}\text { Strain } \\
\text { Compound }\end{array}$ & OFA & TFA & ATFA* & $\begin{array}{c}50-63 / \mathbf{I} \\
\text { OFA }\end{array}$ & $\begin{array}{c}50-63 / \mathbf{I I} \\
\text { TFA }\end{array}$ & $\begin{array}{c}50-63 / I I P \\
\text { ATFA* }\end{array}$ \\
\hline & & & Inh & oitory cone & entration $\dagger$ & \\
\hline Folic acid antagonists & & & & & & \\
\hline Pyrimethamine & 0.175 & 0.25 & 30 & $>\mathbf{3 0 0}$ & $>\mathbf{3 0 0}$ & $>\mathbf{3 0 0}$ \\
\hline Diaveridine & 0.008 & 0.02 & - & $>100$ & $>100$ & - \\
\hline Amethopterin & $0 \cdot 000013$ & 0.028 & - & $0 \cdot 001$ & 0.0039 & - \\
\hline 2,4-Diamino- & & & & & & \\
\hline 6,7-dimethylpteridine & $\mathbf{2} \cdot \mathbf{3}$ & $\mathbf{2 \cdot 8}$ & - & $>100$ & $>100$ & - \\
\hline 6,7-dimethylpyrido-(2,3-d)-pyrimidine & - & 0.42 & - & - & $\mathbf{2 8}$ & 一 \\
\hline 6-ethylquinazoline & - & $0 \cdot 10$ & - & - & 100 & - \\
\hline Thymine antagonists & & & & & & \\
\hline 5-Aminouracil & - & 100 & 145 & - & 21 & 18 \\
\hline 5-Bromouracil & - & 300 & $>\mathbf{3 0 0}$ & - & 30 & 60 \\
\hline 6-Azathymine & - & 300 & $>\mathbf{3 0 0}$ & - & 180 & $>300$ \\
\hline Purine antagonists & & & & & & \\
\hline 6-Mercaptopurine & $23 \cdot 5$ & $22 \cdot 0$ & 165 & $22 \cdot 6$ & 25 & 135 \\
\hline 8-Azaguanine & - & 0.005 & $0 \cdot 125$ & - & 0.006 & $2 \cdot 4$ \\
\hline 2,6-Diaminopurine & $0 \cdot 24$ & 0.06 & $>\mathbf{3 0 0}$ & $10 \cdot 5$ & $0 \cdot 13$ & $>500$ \\
\hline 6-Methylpurine & $2 \cdot 15$ & - & $7 \cdot 4$ & $7 \cdot 0$ & - & 245 \\
\hline Uracil antagonist & & & & & & \\
\hline 6-Azauracil & $1 \cdot 25$ & $1 \cdot 6$ & $0 \cdot 33$ & $1 \cdot 45$ & $2 \cdot 8$ & $0 \cdot 28$ \\
\hline
\end{tabular}

The thymine-requiring mutants exhibited sensitivities to thymine antagonists greater than those of the wild types. The Lactobacillus casei mutants were more sensitive than the wild type to antagonists such as 5-aminouracil, 5-bromouracil and azathymine, while the Proteus mutant, 49-210/II, showed sensitivity to 5-bromouracil, 5-aminouracil, dithiothymine and 2-thiothymine. The effects of the thymine antagonists could be blocked by thymine in the thymineless strains of both Proteus vulgaris and $L$. casei.

The increased purine requirement of strain 50-63/II $\mathrm{p}$ did not give rise to increased sensitivity to purine antagonists. This is in contrast to the above results with the thymine-requiring strains.

Although the wild type and mutant strains of Proteus do not require purines or pyrimidines for growth (with the exception mentioned), purines (adenine, guanine, xanthine, hypoxanthine) and pyrimidines (uracil, cytosine) block the effects of their respective antagonists (6-mercaptopurine, 8-azaguanine, 6-methylpurine, azauracil and isobarbituric acid). Representative data are given in Table 6.

In order to determine whether sensitivity (or resistance) to diaveridine and SD were related, Proteus vulgaris was passaged repeatedly in diaveridine-containing 
Table 5. Cross-resistance with strains of Proteus vulgaris

\author{
Strain \\ Incubation period (hr) \\ Compound
}

Folic acid antagonists

2,4-Diamino-

5-(3',4'-dimethoxybenzyl) pyrimidine

6-ethylquinazoline

5 - $\left(3^{\prime}, 4^{\prime}\right.$-dimethoxyphenoxy) pyrimidine

5-(3',4'-dimethoxy-5'-bromobenzyl) pyrimidine

5 -(3',4',5'-trimethoxybenzyl) pyrimidine

7- $n$-propylpyrido (2,3-d) pyrimidine

5-p-chlorophenyl-6-ethylpyrimidine

Amethopterin

Sulphadiazine (sD)

Thymine antagonists

5-Aminouracil

5-Bromouracil

Dithiothymine

2-Thiothymine

Purine antagonists

6-Mercaptopurine

8-Azaguanine

2,6-Diaminopurine

Thioguanine

6-Methylpurine

Uracil antagonists

6-Azauracil

Isobarbituric acid

Dithiouracil

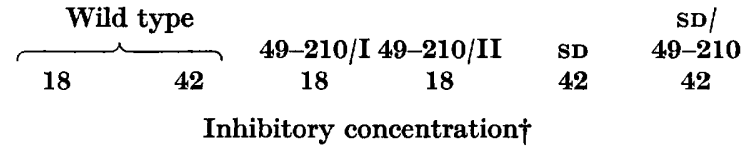

\begin{tabular}{|c|c|c|c|c|}
\hline 34 & - & 16 & 13.5 & - \\
\hline$>\mathbf{3 0 0}$ & 一 & $>300$ & $11 \cdot 0$ & - \\
\hline$>300$ & - & $>300$ & $10 \cdot 0$ & - \\
\hline$>\mathbf{3 0 0}$ & - & $>\mathbf{3 0 0}$ & $1 \cdot 6$ & 一 \\
\hline$>\mathbf{3 0 0}$ & $>\mathbf{3 0 0}$ & 160 & $>\mathbf{3 0 0}$ & $>300$ \\
\hline 30 & 100 & 7 & 15 & 300 \\
\hline$>500$ & $>500$ & 500 & 35 & $>300$ \\
\hline 300 & - & 300 & $>\mathbf{3 0 0}$ & - \\
\hline 11 & $>\mathbf{3 0 0}$ & 13 & $12 \cdot 5$ & 50 \\
\hline $1 \cdot 3$ & $>100$ & 1.0 & $2 \cdot 4$ & $>100$ \\
\hline 3.7 & $>\mathbf{3 0 0}$ & $3 \cdot 7$ & 4.0 & $>300$ \\
\hline $30 \cdot 0$ & - & 100 & $>100$ & \\
\hline
\end{tabular}

$\dagger$ Inhibitory concentration in $\mu \mathrm{g} . / \mathrm{ml}$. giving $\mathbf{5 0} \%$ inhibition.

Table 6. Reversal studies with Proteus vulgaris

\begin{tabular}{cccc}
$\begin{array}{c}\text { Metabolite }(\mu \mathrm{g} . / \mathrm{ml} .) \\
\text { Guanine }\end{array}$ & \multicolumn{3}{c}{$\begin{array}{c}\text { Antimetabolite }(\mu \mathrm{g} . / \mathrm{ml} .) \\
\text { 8-Azaguanine }\end{array}$} \\
$\overbrace{0}$ & 100 & 1000 \\
0 & 80 & 33 & 6 \\
1 & $\mathbf{7 6}$ & 52 & 42 \\
10 & $\mathbf{7 8}$ & $\mathbf{7 8}$ & 78
\end{tabular}

\begin{tabular}{cccccr} 
Uracil* & \multicolumn{5}{c}{ 6-Azauracil } \\
\cline { 2 - 6 } 0 & 0 & $0 \cdot 3$ & 1 & 3 & 10 \\
1 & 75 & 74 & 35 & 2 & 2 \\
3 & 76 & - & - & 66 & 26 \\
10 & 76 & - & - & 70 & 56 \\
& 74 & - & - & 77 & 78
\end{tabular}

* Uridine, uridylic acid, cytosine, cytidine and cytidylic acid gave effects equivalent to those of uracil on a molar basis. The numerical values in the table refer to turbidity as described under Methods. 
medium at several concentrations of the drug and the sensitivities of the derived strains to diaveridine and to sulphadiazine are compared with those of wild type Proteus and strains sD/49-210 (Table 7). The diaveridine-resistant strains were all more sensitive to sulphadiazine than the wild strain by a factor of 2 to 3 . Conversely, the sulphadiazine-resistant strain was approximately 4 times as sensitive as the wild strain to diaveridine.

Table 7. Sensitivities of strains of Proteus vulgaris to sulphadiazine and diaveridine

\begin{tabular}{lrc}
\multicolumn{2}{c}{ Strain } & \multicolumn{2}{c}{ Inhibitory concentration* } \\
Diaveridine & Sulphadiazine \\
Wild type & $4 \cdot 2$ & $\mathbf{8 \cdot 6}$ \\
49-210/10 & $14 \cdot 0$ & $4 \cdot 6$ \\
$49-210 / 30$ & $130 \cdot 0$ & $\mathbf{3 \cdot 8}$ \\
$49-210 / 100$ & $225 \cdot 0$ & $3 \cdot 0$ \\
$49-210 / 300$ & $>1000 \cdot 0$ & $4 \cdot 7$ \\
SD & $1 \cdot 1$ & $530 \cdot 0$ \\
SD $/ 49-210$ & $300 \cdot 0$ & $300 \cdot 0$
\end{tabular}

* Inhibitory concentration in $\mu \mathrm{g} . / \mathrm{ml}$. giving $50 \%$ inhibition after $42 \mathrm{hr}$ growth.

Table 8. Effect of combinations of sulphadiazine and diaveridine on growth of Proteus vulgaris mutants

\begin{tabular}{|c|c|c|c|c|c|}
\hline \multirow[b]{2}{*}{ Strain } & \multirow{2}{*}{$\begin{array}{c}\text { Incubated } \\
(\mathrm{hr})\end{array}$} & \multicolumn{2}{|c|}{ Inhibitory concentrations $\dagger$} & \multicolumn{2}{|c|}{$\begin{array}{l}\text { Potentiative combinations } \\
\text { Sulphadiazine Diaveridine }\end{array}$} \\
\hline & & Sulphadiazine & Diaveridine & f.i.c. & f.i.c. \\
\hline Wild type & 18 & $\mathbf{2 \cdot 5}$ & $\mathbf{2 \cdot 2}$ & 0.09 & $0 \cdot 12$ \\
\hline Wild type* & 18 & $\mathbf{3} \cdot \mathbf{3}$ & $2 \cdot 5$ & 0.15 & 0.09 \\
\hline Wild type & 42 & 4.5 & $5 \cdot 4$ & 0.18 & 0.03 \\
\hline $49-210 / \mathrm{I}$ & 18 & $1 \cdot 3$ & $300 \cdot 0$ & $0 \cdot 27$ & 0.15 \\
\hline $49-210 / \mathrm{II} *$ & 18 & $1 \cdot 2$ & $500 \cdot 0$ & 0.38 & 0.07 \\
\hline sD & 42 & $105 \cdot 0$ & 0.82 & 0.18 & 0.05 \\
\hline $\mathrm{SD} / 49-210$ & 42 & $30 \cdot 0$ & $400 \cdot 0$ & $0 \cdot 38$ & 0.12 \\
\hline
\end{tabular}

$\dagger$ Inhibitory concentration in $\mu \mathrm{g} . / \mathrm{ml}$. giving $\mathbf{5 0} \%$ inhibition.

$\$$ f.i.c. fractional inhibitory concentration: = portion of inhibitory concentration required for $50 \%$ inhibition when sulphadiazine and diaveridine are used in combination at optimum ratio.

* Grown in the presence of thymine, $1 \mu \mathrm{g} . / \mathrm{ml}$, of medium.

The potentiation in combinations of diaveridine and sulphadiazine with respect to the growth of wild-type Proteus has been reported previously (Elion et al. 1960). Table 8 shows the results of experiments designed to determine the magnitude of the potentiative effects in the resistant strains. When combinations of diaveridine and sulphadiazine were tested, potentiation (as measured by the graphical method previously reported, Elion, Singer \& Hitchings, 1954) was still observed, but resistance was reflected in the higher concentrations of drug required to inhibit the growth of the mutants.

\section{DISCUSSION}

It is apparent that one can isolate a variety of strains of Lactobacillus casei and Proteus vulgaris which are resistant to folate antagonists but differ with respect to related biochemical alterations (cf. Hutchison, 1958). The character of the strain one finally obtains depends to a considerable extent on the nutrients which are 
available in the medium. Thus the thymine-less mutants could not have been isolated from a medium which lacked thymine, but these prevail when this nutrilite is available. The biochemical changes which accompany resistance to a drug, therefore, reflect the opportunities available and are not determined solely by the nature of the drug.

The reciprocal (collateral) sensitivities between the sulphonamide- and pyrimidineresistant strains were unexpected. However, this finding is not necessarily inconsistent with the view (Hitchings, 1960, 1961) that these two types of antimetabolite affect steps sequentially arranged on the identical metabolic pathway. When resistance involves altered enzymes, as appears frequently to be the case with sulphonamide resistance (Wolf \& Hotchkiss, 1963; Pato \& Brown, 1963), and recently has been reported in amethopterin resistance (Sirotnak et al. 1963), the reaction would be expected to be less efficient than that catalyzed by the normal enzyme. Such an enzyme might give rise to suboptimum quantities of products, and cells containing it would consequently show increased sensitivity to specific antimetabolites. Such strains might resemble those which were exposed to a metabolite:antimetabolite mixture containing the metabolite in a quantity just sufficient to restore growth. When a second, biochemically related, antimetabolite was introduced into such a system, the organisms were hypersensitive to it, and the effects of the first antimetabolite were nullified completely only when a very high metabolite:antimetabolite ratio was reached (Elion, Singer \& Hitchings, 1958; Singer, Elion \& Hitchings, 1959).

The end-product-requiring strains may derive some advantage through the sparing of folic acid-containing coenzymes. Whether this involves the deletion of one or more of the one-carbon transferases remains to be determined. In thyminerequiring strains, one would expect a reduction in the total tetrahydrofolate requirement by the amount necessary for thymidylate synthetase. Moreover, dihydrofolate reductase would be spared by the amount required for renewal of the 5,10-methylenetetrahydrofolate that is oxidized each time a molecule of thymidylate is synthesized.

With Lactobacillus casei, the mechanisms for the incorporation of exogenous thymine and purine exist in the wild type, since growth can be obtained in the absence of folic acid when these nutrilites are proffered. Its relative inability to use thymidine and thymidylate may reflect a dephosphorylation of thymidylate and a deficiency in thymidine kinase. The wild strain of Proteus vulgaris, however, is unable to incorporate exogenous thymine, and thus the drug-resistant mutant appears to have acquired one or more functions which are not present in the parent. Since this mutant uses thymine, thymidine and thymidylic acid essentially equally well, one might speculate that the enzymes necessary for their incorporation exist in the wild type, and that altered permeability permits their entrance into the mutant. With both organisms, the thymine heterotrophs are more sensitive to thymine antagonists than are the parent strains. However, the sensitivities of purine-requiring mutants to purine antagonists were essentially unaltered from those of the wild types.

Thymine-requiring strains of Lactobacillus casei (Anton \& Nichol, 1959) and Escherichia coli (Okada, Yanagisawa \& Ryan, 1960) have been obtained from amethopterin-inhibited cultures. However, the difficulties with regard to cellular transport of the structural analogues into folate auxotrophs, and the relative ease 
with which the small molecule antagonists are assimilated (Wood, Ferone \& Hitchings, 1961) make the latter more useful inhibitors for the majority of microorganisms.

\section{REFERENCES}

Anton, A. H. \& Nichol, C. A. (1959). Thymine-requiring variant of Lactobacillus casei. J. Bact. 77, 117.

Balis, M. E., Levin, D. H., Brown, G. B., Elion, G. B., VanderWerfy, H. \& Hirchings, G. H. (1952). The incorporation of exogenous purines into pentose nucleic acid by Lactobacillus casei. J. biol. Chem. 196, 729.

Cohen, S. S. \& BARner, H. D. (1954). Studies on unbalanced growth in Escherichia coli. Proc. natn Acad. Sci., U.S.A. 40, 885.

Elion, G. B., Singer, S. \& Hitchings, G. H. (1953). The purine metabolism of a 6-mercaptopurine-resistant Lactobacillus casei. J. biol. Chem. 204, 35.

Elion, G. B., Singer, S. \& Hitchings, G. H. (1954). Antagonists of nucleic acid derivatives. VIII. Synergism in combinations of biochemically related antimetabolites. J. biol. Chem. 208, 477 .

Elion, G. B., Singer, S. \& Hitchings, G. H. (1956). An 8-azaguanine-resistant strain of Lactobacillus casei. Fed. Proc. 15, 248.

Elion, G. B., Singer, S. \& Hitchings, G. H. (1958). Effects of metabolites on inhibitory activities of synergistic combinations of antimetabolites. Fed. Proc. 17, 216.

Elion, G. B., Singer, S. \& Hitchings, G. H. (1960). Potentiation in combinations of three biochemically related antimetabolites. Antibiot. Chemotherapy, 10, 556.

Elion, G. B., VanderWerff, H., Hitchings, G. H., Balis, M. E., Levin, D. H. \& Brown, G. B. (1953). Purine metabolism of a diaminopurine-resistant strain of Lactobacillus casei. J. biol. Chem. 200, 7.

Friedkin, M. (1963). Enzymatic aspects of folic acid. Annu. Rev. Biochem. 32, 185.

Hrtchings, G. H. (1960). Pyrimethamine. The use of an antimetabolite in the chemotherapy of malaria and other infections. Clin. Pharmac. Ther. 1, 570.

Hrtchings, G. H. (1961). A biochemical approach to chemotherapy. Trans. N.Y. Acad. Sci. 23, 700.

Hitchings, G. H. \& Burchall, J. J. (1965). Inhibition of folate biosynthesis and function as a basis for chemotherapy. Advanc. Enzymol. 27, 417.

Hitchings, G. H., Sivger, S. \& Elion, G. B. (1958). Nutrition and drug resistance. In Abstracts of Communications. IV Int. Congr. Biochem. 1958, Sect. 8, p. 98.

Hitchings, G. H., Falco, E. A., VanderWerfy, H., Russell, P. B. \& Elion, G. B. (1952). Antagonists of nucleic acid derivatives. VII. 2,4-diaminopyrimidines. J. biol. Chem. 199, 43.

Hitchings, G. H., Elion, G. B., Falco, E. A., Russell, P. B., Sherwood, M. B. \& VANDERWerFF, H. (1950). Antagonists of nucleic acid derivatives. I. The Lactobacillus casei model. J. biol. Chem. 183, 1.

Huennekens, F. M. \& Osborn, M. J. (1959). Folic acid coenzymes and one carbon metabolism. Advanc. Enzymol. 21, 369.

Hutchison, D. J. (1958). Metabolism of resistant mutants of Streptococcus faecalis. I. Isolation and characterization of the mutants. Cancer Res. 18, 214.

Okada, T., Yanagisawa, K. \& Ryan, F. J. (1960). Elective production of thymine-less mutants. Nature, Lond. 188, 340.

Pato, M. L. \& Brown, G. M. (1963). Mechanisms of resistance of Escherichia coli to sulfonamides. Arch. Biochem. Biophys. 103, 443.

Roth, B., Falco, E. A., Hirchings, G. H. \& Bushby, S. R. M. (1962). 5-Benzyl-2, 4-diaminopyrimidines as antibacterial agents. I. Synthesis and antibacterial activity in vitro. J. mednl pharm. Chem. 5, 1103.

Singer, S., Elion, G. B. \& Hitchings, G. H. (1957). A study of a diaminopyrimidine resistant strain of Proteus vulgaris. Bact. Proc. 115.

Singer, S., Euion, G. B. \& Hitchings, G. H. (1958). Induction of an absolute thymine requirement in Lactobacillus casei. Bact. Proc. $12 \%$. 
Singer, S., Elion, G. B. \& Hitchings, G. H. (1959). The effects of combinations of metabolites on inhibitory activities of synergistic combinations of antimetabolites. Bact. Proc. 114.

Sirotnak, F. M., Donati, G. J. \& Hutchison, D. J. (1963). Folic acid derivatives synthesized during growth of Diplococcus pneumoniae. J. Bact. 85, 658.

Szybalski, W. \& Bryson, V. (1952). Genetic studies on microbial cross resistance to toxic agents. I. Cross resistance of Escherichia coli to fifteen antibiotics. J. Bact. 64, 489.

Worf, B. \& Hotchkiss, R. D. (1963). Genetically modified folic acid synthesizing enzymes of Pneumococcus. Biochemistry, 2, 145.

Wood, R. C., Ferone, R. \& Hrtchings, G. H. (1961). The relationship of cellular permeability to the degree of inhibition by amethopterin and pyrimethamine in several species of bacteria. Biochem. Pharmac. 6, 113. 